\title{
Rapid Identification of Mycobacterium avium subsp. avium from MB/BacT Bottles Using PCR
}

\author{
Andra-Cristina BOSTĂNARU*, Mihai MAREŞ, Aurelian Sorin PAȘCA, Gheorghe SAVUȚA \\ ${ }^{1)}$ Department of Public Health, Faculty of Veterinary Medicine, University of Agriculture Sciences and \\ Veterinary Medicine "Ion Ionescu de la Brad", Iaşi, Romania \\ *Corresponding author: andrutza_mvet@yahoo.com
}

Bulletin UASVM Veterinary Medicine 73(1) / 2016,

Print ISSN 1843-5270; Electronic ISSN 1843-5378

DOI:10.15835/buasvmcn-vm: 10478

\begin{abstract}
The mycobacterial infections caused by strains of Mycobacterium avium complex (MAC) in animals and humans are still present. Mycobacterium avium subsp. avium (MAA) is the etiologic agent of avian tuberculosis, a chronic contagious disease described in a wide variety of domestic and wild bird species. The aim of this study was to evaluate the advantage of using the MB/BacT bottles in an automated system and PCR method for rapid identification of the etiologic agent of tuberculosis in hens. The culture samples isolates from hens in the MB/BacT automated system and PCR amplification of insertion element (IS1245, IS901) it was a faster and specific method.
\end{abstract}

Keywords: IS1245, IS901, MB/BacT, Mycobacterium avium subsp. avium

\section{INTRODUCTION}

The importance of mycobacterial infections caused by strains of Mycobacterium avium complex (MAC) in animals and humans is continuously increasing (Falkinham, 1996; Inderlied et al., 1993; Pavlik et al., 2000).

Mycobacterium avium species consists of four subspecies: M. avium subsp. avium (MAA), M. avium subsp. hominissuis (MAH), M. avium subsp. silvaticum (MAS), and M. avium subsp. paratuberculosis (MAP) (Mijs et al., 2002; OIE, 2014; Thorel et al., 1990), in turn classified into the $\mathrm{S}$ (sheep) and C (cattle) types. These subspecies, although closely related are different, each with specific pathogenetic and host range characteristics, ranging from environmental opportunistic bacteria that cause infections in swine and immunocompromised patients to pathogens of birds and ruminants (Laura Rindi and Grazelli, 2014; Thorel et al., 1990).

Avian tuberculosis is one of the most important diseases that affects most of the birds (Mansour et al., 2013; OIE, 2014; Tell et al., 2001;
Van Dar Heyden et al., 1997). Avian tuberculosis is the most frequently produced by infection with Mycobacterium avium subsp. avium (serotypes 1, 2 and 3, containing specific gene segment IS901 and nonspecific segment IS1245) and less frequently by M. genavense (Dvorska et al., 2007; Fulton and Sanchez, 2008; Guerrero et al., 1995; Mansour et al., 2013; OIE, 2014; Pavlik et al., 2000; Tell et al., 2001). Avian tuberculosis is caused by other two members of $M$. avium complex: M. avium subsp. hominissuis (serotypes 4-6, 8-11 and 2; lacking gene segment IS901 and containing segment IS1245) and M. intracellulare (serotypes 7, 12-20 and 22-28; lacking both gene segments IS901 and IS1245) and by M. intracellulare, M. scrofulaceum, $M$. fortuitum and other potentially pathogenic mycobacterial species (OIE, 2014).

MAA is characterised by the presence of 2 to 17 copies of the IS901 insertion sequence (Dvorska et al., 2003; Inglis et al., 2003; Kaevska et al., 2010) and a single copy of IS1245 (Johansen et al., 2007). IS901 has only been detected in $M$. avium strains with serotypes 1, 2 and 3 (Pavlik et 
al., 2000; Ritacco et al., 1998) which is considered more pathogenic to birds than other serotypes (OIE, 2014; Tell et al., 2001).

MAA remains the most prevalent agent of avian tuberculosis in the domestic hens (Gonzalez et al., 2002; Kaevska et al., 2010; Shitaye et al., 2008). MAA was isolated as non-tuberculous mycobacteria (NTM) species from opportunistic infections in humans (Kaevska et al., 2010; Pavlik et al., 2000).

It is considered that under favourable conditions, all species of birds are susceptible to MAA infection, although among domestic birds, hens (Gallus domesticus) are the most susceptible species (Shitaye et al., 2008).

One of the most important ways to reduce the incidence of infection with mycobacteria is by using a rapid diagnosis, particularly for high numbers of bacilli in sample who pose a greater risk of transmission (Gil-Setas et al., 2004).

Considering that the conventional culture methods such as the use of Lowenstein Jensen (LJ) medium which requires 3 to 6 weeks for its isolation, plus an additional 1 to 2 weeks for its identification and that we still face with mycobacterial infections is necessary a rapid detection (Naveen and Basavaraj, 2012).

According to the Centers for Disease Control (1995) recommendations for mycobacteriology laboratories, the use of liquid medium has become one of the main diagnostic techniques. However, many laboratories have not introduced radiometric methods because they require expensive specialised equipment, qualified personnel and the safe disposal of radioactive wastes (Gil-Setas et al., 2004). The combined use of a liquid and a solid medium has been recommended (Gil-Setas et al., 2004; Metchock et al., 1999).

Considering these aspects, there is a need of a culture method that is reliable and which has a short turnaround time. All methods have their own advantages and disadvantages, starting from the LJ medium to the present and speedy automated methods like the MB/BACT device (Adler et al., 2005; Naveen and Basavaraj, 2012). MB/ BACT is a safer and rapid method because is an automatically method which contains liquid media and not requires any radioactive material (Naveen and Basavaraj, 2012). One of the disadvantages for culture in liquid medium is that it does not provide visible colonies (Mirovic and Lepsanovic, 2002).
This paper presents the results performed at the hans with clinical tuberculosis, that were diagnosticated in the Faculty of Veterinary Medicine in Iasi.

For the detection of the subspecies Mycobacterium avium subsp. avium in tissues of hens it was used an MB/BacT automated system and PCR assay with the purpose to facilitated a rapid decision regarding the tuberculosis outbreak.

\section{MATERIALS AND METHODS}

A total of nine birds from species Gallus domesticus were examined. All the hens were originated from different households in Iași county.

The health status of the hens was evaluated for clinical signs of diarrhoea, emaciation and weakness that have evolved with a sporadic death. During the dissection, the organs were examined for the presence of tuberculous lesions.

Gross examination and histopathology. The tissue samples from naturally dead hens were rapidly examined for the presence of tuberculous lesions. The organs which had tuberculous lesions (liver, spleen and intestine), were formalin fixed, embedded in paraffin blocks and stained by ZiehlNeelsen (ZN) technique for the presence of acidfast bacilli (AFB) and hematoxylin eosin methylene blue (HEA) for histological evaluation of the granulomatous lesions. Histological samples were examined with a microscope Leica ICC50 HD using a 1000 magnification under oil immersion and capturing images with the Acquire Leica Software system.

Isolation of mycobacteria. The smears prepared from samples with typical lesions were stained according to the $\mathrm{ZN}$ technique for the presence of acid-fast bacilli (AFB).

For culture examination, the hens samples were concentrated and decontaminated using the modified Petroff's technique with $4 \%$ sodium hydroxide (Allen and Baker, 1968).

The inoculum was digested and planted into three tubes slants of LJ medium and Herrolds's egg yolk media (HEYM) with Mycobactin J for each specimen. For shorter incubation times was used cultivation on MB/BacT bottles (bioMérieux, France), incubated at automated MB/BacT system (Organon Teknika). The MB/BacT bottles were analyzed every 10 minutes using the standard BacT/ALERT software. Incubation was performed 
at $37^{\circ} \mathrm{C}$ for two months. The positive culture tubes were examined using $\mathrm{ZN}$ staining to observe a possible contamination and to confirm the presence of AFB.

Identification of isolates. The detection method was based on a specific and sensitive PCR for insertion element which enabled identification of MAA.

DNA was extracted from single colonies and was prepared for mycobacteria identification by performing the heat shook.

Mycobacterium avium subsp. avium CECT 7407 was used as the reference strain. All positive isolates, both in the solid and liquid medium, were examined by the PCR method for detection of IS1245, IS901 a specific insertion sequences for $M$. avium subspecies, using primers (Tab. 1) according to Miller et al., (1999).

The PCR assay was performed using the kit $\mathrm{IQ}^{\mathrm{TM}}$ Supermix (BIO-RAD). IQ ${ }^{\mathrm{TM}}$ Supermix contains the following reagents dNTPs, iTaq DNA polymerase, $6 \mathrm{mM} \mathrm{MgCl}_{2}$ and stabilizers. For IS901 and IS1245 PCR were used $5 \mu \mathrm{l}$ of DNA template and $45 \mu \mathrm{l} \mathrm{PCR} \mathrm{mix:} 15 \mu \mathrm{l}$ water; $20 \mu \mathrm{l}$ IQ supermix $1 \mathrm{x}, 5 \mu \mathrm{l}$ forward and reverse primer $20 \mathrm{pmol}$.

The amplification conditions for primers IS901 and IS 1245 were as follows: $10 \mathrm{~min}$ at $94^{\circ} \mathrm{C}$; 30 cycles of $60 \mathrm{~s}$ at $94^{\circ} \mathrm{C}$, primer annealing at $62^{\circ} \mathrm{C}$ for $30 \mathrm{~s}$, elongation $1 \mathrm{~min}$ at $72^{\circ} \mathrm{C}$ using a thermocycler (model MJ Mini Thermal Cycler, BIO-RAD), with a $10 \mathrm{~min}$ final extension at $72^{\circ} \mathrm{C}$. Amplification was analyzed by electrophoresis with $5 \mu$ l sample in $1 \%$ agarose and staining with ethidium bromide.

\section{RESULTS AND DISCUSSIONS}

Clinic signs and pathological lesions. In most cases, the infected birds showed no clinical signs, but they were lethargic and emaciated. At four hens it was observed a deformation of the breast bone. Affected birds were older than one year.

At post-mortem examination was observed severe lesions in the intestinal tract with typical caseous lesions in the liver and spleen. Most often these lesions were present in the liver, less frequently in the spleen and sporadically in the small intestine. The liver and spleen were

Tab. 1.Oligonucleotide primers used for PCR amplification of mycobacterial DNA

\begin{tabular}{ccccc}
\hline Organism(s) & $\begin{array}{c}\text { Insertion } \\
\text { sequence }\end{array}$ & $\begin{array}{c}\text { Amplicon } \\
\text { size }(\mathrm{bp})\end{array}$ & Sequence (5'-3') & Source \\
\hline $\begin{array}{c}\text { M. avium } \\
\text { complex }\end{array}$ & IS1245 & 427 & $\begin{array}{c}\text { GCCGCCGAAACGATCTAC } \\
\text { AGGTGGCGTCGAGGAAGA }\end{array}$ & Miller et al. \\
\hline $\begin{array}{c}\text { M. avium } \\
\text { subsp. avium }\end{array}$ & IS901 & 252 & $\begin{array}{c}\text { GCAACGGTTGTTGCTGAAA } \\
\text { TGATACGGCCGGAATCGCGT }\end{array}$ & Miller et al. \\
\hline
\end{tabular}
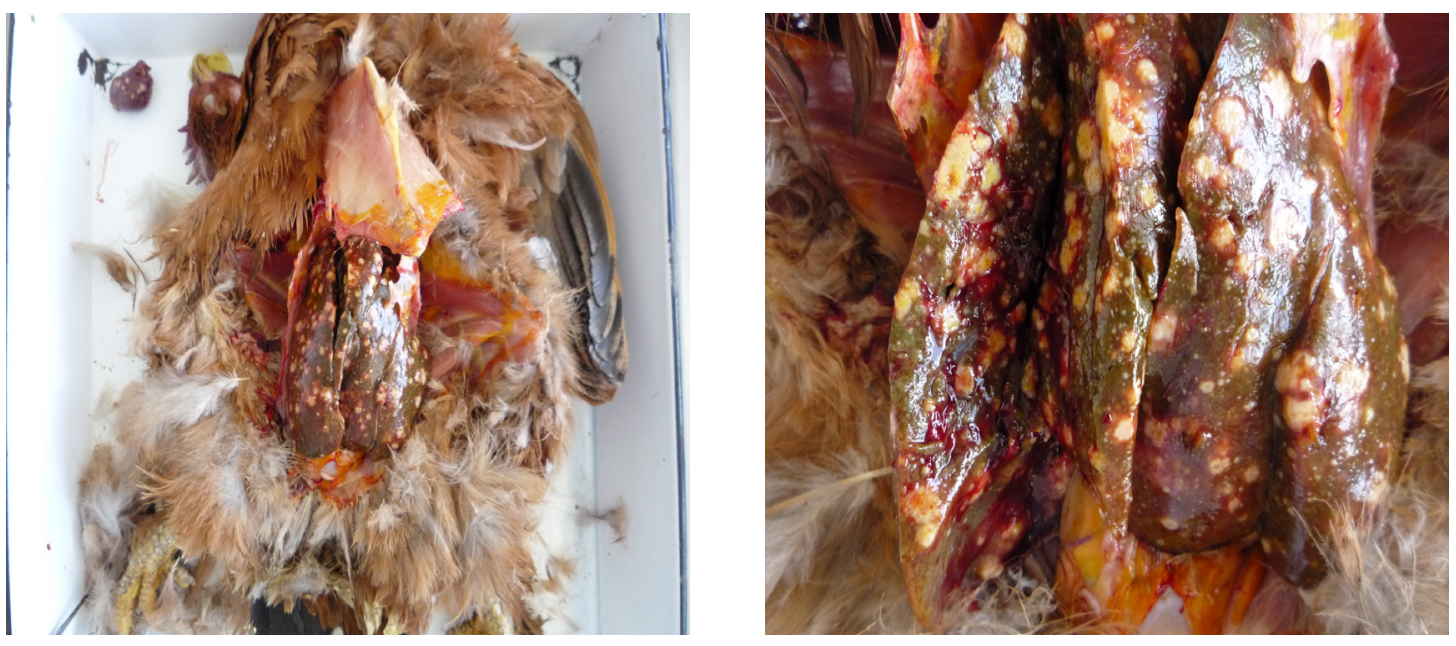

Fig. 1. Hens. Nodular granulomatous lesions in the liver 
hypertrophied, exceeding the normal size, with increased friability and with the presence of granulomatous nodules of considerable size (Fig.1).

Gross examination and histopathology. The histopathological lesions in the liver, spleen and intestine were characteristic with infection of mycobacteria (avian tuberculosis), with presence of the granulomas in various stages of development and with the observation of three types of nodules.

It was found both the granuloma which contain necrosis (Fig. 2) as well as the non-necrotic granuloma.

The formation of the granuloma was characterized by the presence of multinucleated giant cells with nuclei arranged like a horseshoe (Langhans giant cell) and for the mature granuloma it was observed a caseous central necrosis (Fig. 2). The central caseum was surrounded by epithelioid macrophages, Langhans-type giant cells, peripheral lymphocytes and collagen fibres (fibrosis).

In conclusion, all hens showed lesions with typical granulomas in liver, spleen and intestine, with central necrosis and cellular components of chronic inflammation and with detection of numerous acid-fast bacilli.

The diagnosis of $M$. avium infection is based on clinical signs, postmortem gross lesions, and by observing the acid-fast bacilli in crushed lesions using microscopy, which is sufficient for a positive diagnosis (Fulton and Thoen, 2003; Tell et al, 2001; Kuldeep et al., 2011).

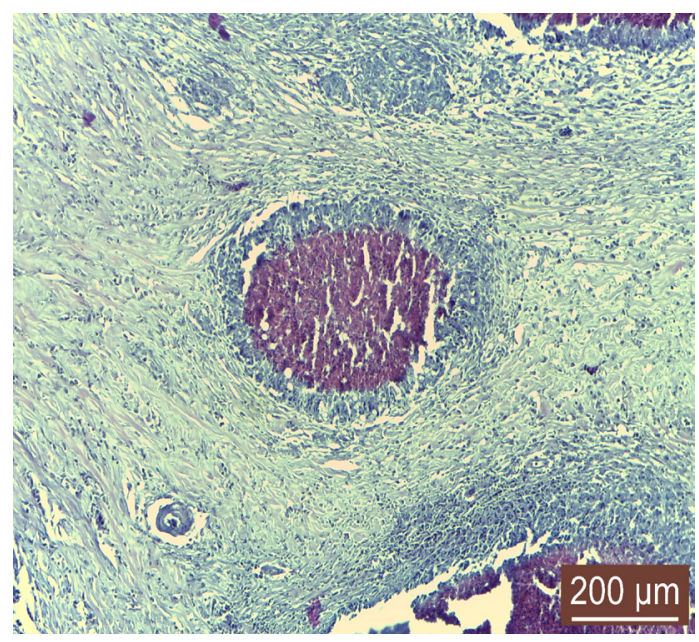

Fig. 2. Hens. Liver. Tuberculous granulomas; HEA

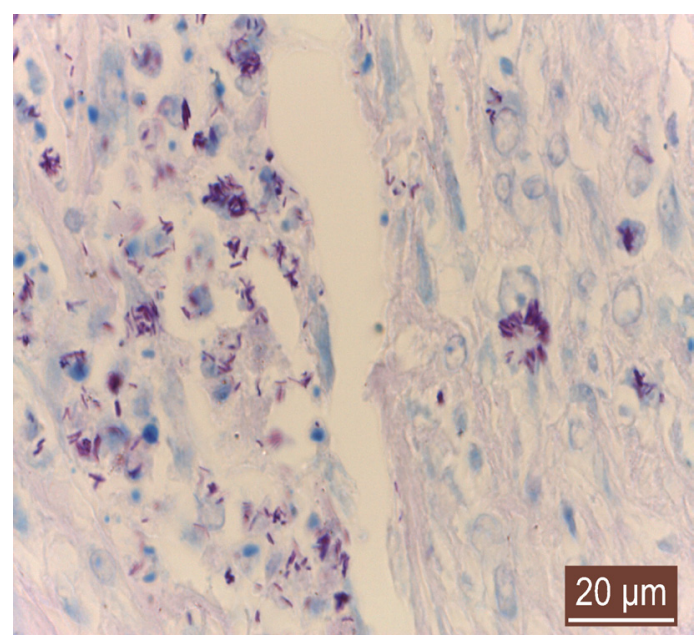

A

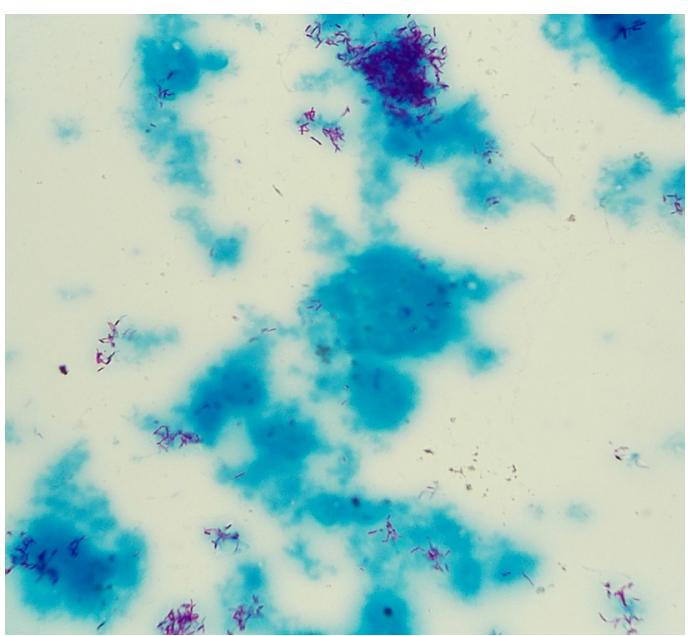

$\mathrm{B}$

Fig. 3. Hens. Spleen. A. - AFB in tissues; B. - AFB in the obtained culture on MB/BacT, ZN 
Culture examination. After culture examination, there obtained isolates from all hens. After incubation there produced typical smooth colonies transparent in both medium. The growth from the cultures were confirmed by ZN staining proving infection with mycobacteria (Fig. 3).

In this study was attempted the feasibility of using $\mathrm{MB} / \mathrm{BacT}$ and $\mathrm{LJ}$ as the primary isolation media for mycobacteria. The two media were compared regarding the number of isolates, the rate of isolation and the mean duration of the isolation. In addition, the isolates from both $\mathrm{MB} /$ BacT and LJ medium was verified by PCR.

At a hen the sample on the LJ medium and HEYM with mycobactin, could not be isolated,

Tab. 2. Comparison of MB/BacT and LJ for duration of isolation and number of isolates of MAA

\begin{tabular}{ccccc}
\hline & \multicolumn{3}{c}{$\begin{array}{c}\text { Duration of } \\
\text { isolation (days) }\end{array}$} & \multicolumn{2}{c}{ Culture positive } \\
\cline { 2 - 5 } No of sample & LJ & MB/BacT & LJ & MB/BacT \\
\hline $\mathbf{1}$ & 6 & 4 & + & + \\
\hline $\mathbf{2}$ & 15 & 8 & + & + \\
\hline 3 & 9 & 6 & + & + \\
\hline $\mathbf{4}$ & 12 & 8 & + & + \\
\hline $\mathbf{5}$ & 20 & 9 & - & + \\
\hline $\mathbf{6}$ & 14 & 8 & + & + \\
\hline $\mathbf{7}$ & 10 & 5 & + & + \\
\hline $\mathbf{8}$ & 11 & 6 & + & + \\
\hline $\mathbf{9}$ & 11 & 5 & + & + \\
\hline $\begin{array}{c}\text { Mean } \\
\text { duration } \\
\text { / No of } \\
\text { isolates }\end{array}$ & 12 & 6.5 & $8 / 9$ & $9 / 9$ \\
\hline
\end{tabular}

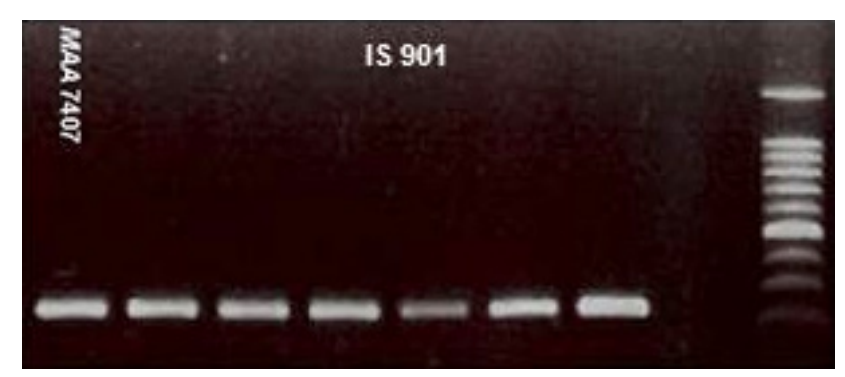

A but in MB/Bact it was observed a positive sample (Tab. 2).

The mean duration of the isolation on LJ and $\mathrm{MB} / \mathrm{BacT}$ was 12 days and 6.5 days respectively (Tab.2). The difference between the LJ medium and the MB/BacT medium was significant. The liquid media in $\mathrm{MB} / \mathrm{BacT}$ proved to be more successful comparative with LJ, because is faster (6.5 days compared to 12 days).

$\mathrm{MB} /$ BacT it proved to be superior to LJ medium in the isolation rate, because it could isolate mycobacteria with 7-10 days earlier (Naveen and Basavaraj, 2012).

PCR identification. All isolated strains obtained at hens on $\mathrm{LJ}$ and MB/BacT medium, were detected by PCR.

The isolates were positive for IS901 and IS1245 PCR, with the visualization of a $252 \mathrm{bp}$ fragment and $427 \mathrm{bp}$ fragment, respectively.

After performing the PCR, all hens ( $\mathrm{n}=9)$ were found positive for both IS1245 and IS901 (Fig. 4), confirming infection with Mycobacterium avium subsp. avium.

According to the results obtained at PCR we can reach to the conclusion that MB/Bact automated system is a faster and also reliable alternative to conventional culture methods.

\section{CONCLUSION}

Diagnosis of MAA (containing IS901) from tissue samples was confirmed using conventional PCR.

The sensitivity and the time to detection were significantly better with MB/BacT than with solid LJ medium. The culture samples isolated from hens in the MB/BacT automated system and PCR amplification of insertion element were rapid and specific methods.

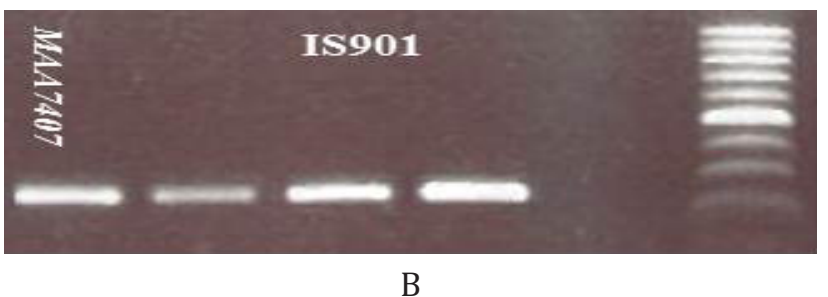

Fig.4. -PCR amplification product of the 252 bp specific fragment from IS901;

IS 901 PCR performed on extract isolated from MB/BacT;

A - samples for 6 hens; B - samples for 3 hens; MAA 7407 - postive control. 
The results indicates that the $\mathrm{MB} / \mathrm{BacT}$ system has great potential: it is a rapid, sensitive, safe, simple-to-use, and an automated method for the isolation of mycobacteria.

Acknowledgments. The authors are thankful to the Department of Bacteriology of Clinical Hospital of Pulmonology Iaşi, for providing MB/BacT an automated system required for conducting the research work. This work was supported from the Project: "Improvement and Development of Human Resources for Research and Innovation by Doctoral School", Contract: POSDRU-CPP107DMI1/5/S/77222; University of Agricultural Sciences and Veterinary Medicine, Iași, Romania.

\section{REFERENCES}

1. Adler H, Straub C , Frel R (2005). Comparison of MB/BacT, Lowenstein -Jensen medium and MB7H10 / 7H11 biplate for recovering Mycobacteria from clinical specimens. Eur J Clin Microbiol infect diseases 24:499-500.

2. Allen BW, Baker FJ (1968). Modified Petroff Technique Treatment of specimens, chapter 3. In : Mycobacteria Isolation, Identification and Sensitivity Testing. Allen BW, Baker FJ, Butterworth and Co, London, 9-16.

3. Centers for Disease Control and Prevention (1995). Essential components of a tuberculosis prevention and control program. Recommendations of the Advisory Council for the Elimination of Tuberculosis. Morbid Mortal Weekly Rep 44(RR-11):1-16.

4. Dvorska L, Matlova L, Ayele WY, Fischer OA, Amemori T, Weston RT (2007). Avian tuberculosis in naturally infected captive water birds of the Ardeideae and Threskiornithidae families studied by serotyping, IS901 RFLP typing, and virulence for poultry. Vet Microbiol 119:366-374.

5. Falkinham JF (1996). Epidemiology of infection by nontuberculous mycobacteria. Clin Microbiol Rev 9:177215.

6. Fulton R, Sanchez S (2008). Tuberculosis. In: YM Saif, HJ Barnes, JR Glisson, AM Fadly, LR McDougald, DE Swayne (Eds.). Disease of poultry, 12th ed, Blackwell Publishing, Iowa State Press , Ames, 940-951.

7. Fulton RM, Thoen CO (2003). Tuberculosis. In: YM Saif, HJ Barnes, JR Glisson, AM Fadly, LR McDougald, DE Swayne (Eds.). Disease of poultry,Iowa State University Press, Ames, 836-844.

8. Gil-Setas A, Torroba L, Fernandez JL, Martinez-Artola L, Olite J (2004). Evaluation of the MB/BacT system compared with Middlebrook 7H11 and LowensteinJensen media for detection and recovery of mycobacteria from clinical specimens. Clinical Microbiology and Infection 10(3):224-228.

9. Gonzalez M, Rodriguez-Bertos A, Gimeno I, Flores JM, Pizarro M (2002). Outbreak of avian tuberculosis in 48-week-old commercial layer hen flock. Avian Diseases 46:1055-1061.

10. Guerrero C, Bernasconi C, Burki D, Bodmer T, Telenti A (1995). A novel insertion element from Mycobacterium avium, IS1245, is a specific target for analysis of strain relatedness. Journal of Clinical Microbiology 33:304-307.

11. Inderlied CB, Kemper CA, Bermudez LE (1993). The Mycobacterium avium complex. Clin Microbiol Rev 6:266-310.

12. Inglis NF, Stevenson K, Heaslip DG, Sharp JM (2003). Characterisation of IS901 integration sites in the Mycobacterium avium genome. FEMS Microbiology Letters 221:39-47.

13. Janice MM, Allen LJ, Jay LE (1999). Polymerase chain reaction identification of Mycobacterium avium in formalin-fixed, paraffin-embedded animal tissues. J Vet Diagn Invest 11:436-440.

14. Johansen TB, Olsen I, Jensen MR, Dahle UR, Holstad G, Djonne B (2007). New probes used for IS1245 and IS1311 restriction fragment length polymorphism of Mycobacterium avium subsp. avium and Mycobacterium avium subsp. hominissuis isolates of human and animal origin in Norway. BMC Microbiology 7-14.

15. Kaevska M, Slana I, Kralik P, Pavlik I(2010). Examination of Mycobacterium avium subsp. avium distribution in naturally infected hens by culture and triplex quantitative real time PCR. Veterinarni Medicina 55(7): 325-330.

16. Kuldeep D, Mahesh M, Ruchi T, Shambhu DS, Deepak K, Shoorvir S, Pradeep MS (2011). Tuberculosis in Birds: Insights into the Mycobacterium avium Infections Veterinary Medicine International Volume, Article ID 712369.

17. Rindi L, Garzelli C (2014). Genetic diversity and phylogeny of Mycobacterium avium. Infection, Genetics and Evolution 21:375-383.

18. Mansour M, Nader M, Saleh E, Kaveh PA (2013). Comparison of four different culture media for growth of Mycobacterium avium subsp. avium isolated from naturally infected lofts of domestic pigeons. 5(4):379-382.

19. Manual of Diagnostic Tests and Vaccines for Terrestrial Animals. Avian tuberculosis (2014).Chapter 2.3.6.:497508. http://www.oie.int/en/international-standardsetting/terrestrial-manual/access-online.

20. Metchock BG, Nolte FS, Wallace RJ (1999). Mycobacterium. In : PR Murray, EJ Baron, MA Pfaller, FC Tenover, RH Yolken (ed.). Manual of clinical microbiology, 7 th ed. ASM Press,Washington, 399-437.

21. Mijs W, De Haas P, Rossau R, Van Der Laan T, Rigouts L, Portaels F, Van Soolingen D (2002). Molecular evidence to support a proposal to reserve the designation Mycobacterium avium subsp. avium to bird-type isolates and M. avium subsp. hominissuis for the human/porcine type of M. avium. Int J Syst Evol Microbiol 52:1505-1518.

22. Miller JM, Jenny AL, Ellingson JL (1999). Polymerase chain reaction identification of Mycobacterium avium in formalin-fixed, paraffin-embedded animal tissues. Journal of Veterinary Diagnostic Investigation 11:436-440. 
23. Mirovic V, Lepsanovic Z (2002). Evaluation of the MB/ BacT System for recovery of mycobacteria from clinical specimens in comparison to Lowenstein-Jensen medium. Clin Microbiol Infect 8:709-714.

24. Naveen G, Basavaraj VP (2012). Comparison of the Lowenstein-Jensen Medium, the Middlebrook $7 \mathrm{H} 10$ Medium and MB/BacT for the Isolation of Mycobacterium tuberculosis (MTB) from Clinical Specimens. Journal of clinical and diagnostic research JCDR 12/ 6(10):17041709.

25. Pavlik I, Svastova P, Bartl J, Dvorska L, Rychlik I (2000). Relationship between IS901 in the Mycobacterium avium Complex Strains Isolated from Birds, Animals, Humans, and the Environment and Virulence for Poultry. Clin Diagn Lab Immunol 7:212-217.

26. Ritacco V, Kremer K, Van Der Laan T, Pijnenburg JEM, De Haas PEW, Van Soolingen D (1998). Use of IS901 and IS1245 in RFLP typing of Mycobacterium avium complex: relatedness among serovar reference strains, human and animal isolates. Int J Tuberculosis Lung Dis 2:242-251.

27. Shitaye JE, Matlova L, Horvathova A, Moravkova M, Dvorska-Bartosova L,Treml F (2008). Mycobacterium avium subsp. avium distribution studied in a naturally infected hen flock and in the environment by culture, serotyping and IS901 RFLP methods. Vet Microbiol 127:155-164.

28. Tell LA, Woods L, Cromie RL (2001). Tuberculosis in birds. Review Science and Technology Office Internationale des Epizooties 20:180-203.

29. Thorel MF, Krichevsky M, Levy-Frebault VV (1990). Numerical taxonomy of mycobactin-dependent mycobacteria, emended description of Mycobacterium avium, and description of Mycobacterium avium subsp. avium subsp. nov., Mycobacterium avium subsp. paratuberculosis subsp. nov., and Mycobacterium avium subsp. silvaticum subsp. nov. Int J Syst Bacteriol 40(3):254260.

30. Van Dar Heyden N (1997). Clinical manifestations of mycobacteriosis in pet birds. Seminars in Avian and Exotic Pet Medicines 6:18-24.

31. Wilson ML, Stone BL, Hildred MV, Reves RR (1995). Comparison of recovery rates for Mycobacteria from BACTEC 12B vials, Middlebrook 7H11- Selective 7H11 biplates and Lowenstein Jensen slants in a public health mycobacteriology laboratory. Jou Clin Micro 2516-18. 